\title{
Association of pre-surgery to pre- radiotherapy lymphocyte counts ratio with disease-free survival in rectal cancer patients receiving neoadjuvant concurrent chemoradiotherapy
}

Hongen $\mathrm{Xu}^{1+}$, Guangxian You ${ }^{2+}$, Minjun Zhang ${ }^{1,3+}$, Tao Song ${ }^{1}$, Haibo Zhang ${ }^{1}$, Jia Yang ${ }^{1}$, Yongshi Jia ${ }^{1}$, Jianming Tang ${ }^{1 *}$ and Xiaodong Liang ${ }^{1 *}$

\begin{abstract}
Background: Colorectal cancer is the fourth most common cancer globally and neoadjuvant concurrent chemoradiotherapy (nCRT) and surgery are the standard treatments for locally advanced colorectal carcinoma. This study investigated the association between dynamic changes in absolute lymphocyte counts (ALCs) and diseasefree survival (DFS) in rectal cancer patients receiving nCRT and identified factors associated with these changes.

Methods: We retrospectively examined 34 patients with locally advanced rectal cancer who received nCRT followed by surgery and adjuvant chemotherapy. The association between ALCs and DFS and that between ALCS and downstaging were analyzed and potential clinical- and treatment-related factors related to dynamic changes in ALCs were subsequently evaluated. The patient eligibility criteria were as follows: pathologically confirmed rectal adenocarcinoma, clinical stages II-III, $\geq 18$ years of age, and so on. Pre-RTL was defined as ALCs obtained before the initiation of nCRT and pre-SL was defined as ALCs obtained before surgery. We measured pre-SL to pre-RTL ratio (pre-SLR), DFS, and ALCs.

Results: The median ALC declined significantly during nCRT. A lower pre-SLR was associated with poorer DFS with statistical significance in Kaplan-Meier ( $p=0.007$ ), univariate regression (hazard ratio [HR] $=6.287,95 \%$ confidence interval [Cl] 1.374-28.781, $p=0.018$ ), and multivariable regression ( $H R=7.347,95 \% \mathrm{Cl} 1.595-33.850, p=0.011)$ analyses. Neither patient characteristics nor treatment-related factors were related to downstaging. The pelvic bone marrow (PBM) volume receiving at least $30 \mathrm{~Gy}$ (V30) was significantly associated with pre-SLR in the univariate (HR $=5.760,95 \%$ Cl 1.317-25.187, $p=0.020)$ and multivariable ( $\mathrm{HR}=5.760,95 \% \mathrm{Cl} 1.317-25.187, p=0.020)$ regression analyses.
\end{abstract}

Limitations: Our study had several limitations. The sample size was small and the study was performed in a selected population, which may limit the generalization of the findings.

Conclusions: Radiotherapy had a profound impact on the change in ALCs. A lower pre-SLR was significantly associated with poorer DFS in rectal cancer patients receiving nCRT. The V30 of PBM was a predictor of pre-SLR.

Keywords: Rectal cancer, Lymphocyte counts, Disease-free survival, Chemoradiotherapy

\footnotetext{
*Correspondence: sjg89891@163.com; Ixdctopone@163.com

${ }^{\dagger}$ Hongen Xu, Guangxian You and Minjun Zhang are listed as co-first authors.

'Department of Radiation Oncology, Cancer Center, Zhejiang Provincial

People's Hospital, People's Hospital of Hangzhou Medical College, No. 158,

Shangtang Road, Xiacheng District, Hangzhou 310014, China

Full list of author information is available at the end of the article
}

(c) The Author(s). 2019 Open Access This article is distributed under the terms of the Creative Commons Attribution 4.0 International License (http://creativecommons.org/licenses/by/4.0/), which permits unrestricted use, distribution, and reproduction in any medium, provided you give appropriate credit to the original author(s) and the source, provide a link to the Creative Commons license, and indicate if changes were made. The Creative Commons Public Domain Dedication waiver (http://creativecommons.org/publicdomain/zero/1.0/) applies to the data made available in this article, unless otherwise stated. 


\section{Background}

The management of local advanced colorectal cancer is timely. Colorectal cancer is the fourth most common cancer globally [1]. Although significant improvements in the treatment of rectal cancer have recently been made, the overall 5-year survival rate for the whole population is dismal, at less than $60 \%$. Compared with adjuvant chemoradiotherapy, neoadjuvant concurrent chemoradiotherapy (nCRT) improves the local control rate and decreases toxicities. nCRT has also been shown to increase the rate of sphincter preservation and improve quality of life $[2,3]$. nCRT and surgery are, therefore, the standard treatments for locally advanced colorectal carcinoma.

A series of studies has shown that the host immune system is significantly associated with the prognosis of patients with colorectal cancer. Tumor-infiltrating lymphocytes $[4,5]$ are associated with the prognosis in colorectal cancer. However, nCRT may have a detrimental effect on host immune function. Among all immune factors, lymphocytes play a key role in host immune function. Circulating lymphopenia has been shown to be a prognostic factor in non-small-cell lung cancer, esophageal cancer, pancreatic carcinoma, and other solid cancers [6]. In rectal cancer, lymphocyte count is both a predictor and a prognostic factor. A high ratio of lymphocytes in white blood cells before treatment is associated with not only higher pathologic complete remission but also better disease-free survival (DFS) and overall survival (OS) in rectal cancer [7]. Similarly, a better pathologic complete response rate was achieved in patients with higher sustained lymphocyte counts at 4 weeks during nCRT [8]. In addition, lymphopenia is associated with prognosis in patients with advanced colorectal cancer receiving chemotherapy [9].

However, the association between DFS and dynamic changes in ALCs is not well documented in the literature. The present study aimed to explore whether DFS is associated with circulating ALCs before chemoradiotherapy (pre-RTL), during nCRT, and before surgery (pre-SL) in patients with locally advanced rectal cancer and to explore the potential clinical and treatment-related factors associated with lymphocyte counts.

\section{Materials and methods Patient selection}

This study was reviewed and approved by the medical ethics committee of Zhejiang Provincial People's Hospital. Informed consent was waived by the board due to the retrospective design of this study. The patient eligibility criteria were as follows: pathologically confirmed rectal adenocarcinoma, clinical stages II-III, $\geq$ 18 years of age, Eastern Cooperative Oncology Group
(ECOG) performance status of $0-1$, baseline complete blood counts and carcino-embryonic antigen (CEA) data available, received nCRT followed by surgery-at our institution, total mesorectal excision (TME) performed with $R_{0}$ resection, no history of previous radiotherapy or chemotherapy, no history of previous or coexisting cancer, and at least three weekly complete blood counts documented during nCRT. ALCs were obtained from complete blood count tests. ALCs obtained before the initiation of nCRT (within 1 week) were defined as pre-RTL, while ALCs obtained before surgery (within 1 week) were defined as pre-SL. The pre-SLR was defined as the ratio of pre-SL to preRTL. The lowest ALC was considered as the lymphocyte nadir during nCRT (W1 to W5). Lymphocyte toxicity was evaluated according to the National Cancer Institute's Common Terminology Criteria for Adverse Events (CTCAE) version 4.0. Downstaging was defined as pathologically staged T0-2 N0 after surgery (yp $0-2 \mathrm{~N} 0$ ).

Patients were followed up every 3 months for 2 years after surgery, every 6 months in the third to fifth year, and then on a yearly basis. Local recurrence was considered as a recurrence within the pelvis (below the fifth lumbar spine L5). New lesions beyond the pelvis were considered distal metastasis. DFS was calculated from the start date of CRT to the date of first documented radiographic failure or death from any cause.

\section{Evaluation of the PBM dose}

The external contour of all the bones within the pelvis was contoured as pelvic bone marrow (PBM) on computed tomography (CT) simulation (5-mm slice thickness), from the bottom of the fourth lumbar vertebra to the bottom of the ischial tuberosities. The dose-volume histogram (DVH) parameters of the PBM were generated using a Pinnacle Treatment Planning System. The V5, V10, V20, V30, and V40 parameters were calculated as the percentages of volumes receiving at least 5, 10, 20, 30, and 40 Gray (Gy), respectively. The $D_{\text {mean }}$ was calculated as the mean dose to the PBM.

\section{Statistical analysis}

Friedman tests were used to compare the ALCs at different time points during nCRT. DFS was calculated (the Kaplan-Meier method) and compared (logrank tests) between groups. The association between clinical data and downstaging was evaluated by Fisher's exact tests. The associations between potential prognostic factors and DFS were evaluated by univariate and multivariate analyses using a Cox regression model. We further analyzed the association between 
clinical data and the pre-SLR level in univariate and multivariate analyses. The multivariate analysis was performed with logistic regression backward stepwise methods, including all of the variables used in univariate analysis. The variables were considered as dichotomous variables. Lymphocyte nadir was dichotomized at $0.5 \times 10^{9}$ cells $/ \mathrm{L}$. All other continuous variables were dichotomized at the median level. All statistical analyses were performed using IBM SPSS Statistics for Windows, version 25.0 (IBM Corp., Armonk, NY). Significant differences were defined as two-sided pvalues of less than 0.05 .

\section{Results}

\section{Patient and treatment characteristics}

A total of 41 patients with pathologically diagnosed rectal cancer were treated in our institution with nCRT between January 2013 and December 2017. Seven patients were excluded from the study for other cancers (two patients), resectable liver metastases (four patients), and incomplete data (one patient). Thirty-four patients with complete clinical data were finally included in the current study. Their characteristics are shown in Table 1. Standard TME was performed in all patients with negative margin $\left(R_{0}\right.$ resection).

\section{nCRT}

Patients were required to undergo CT simulation with contrast. The clinical target volume (CTV) included the gross tumor with a margin of $2-3 \mathrm{~cm}$, mesorectum, presacral lymph nodes, and internal iliac lymph nodes. The $\mathrm{CTV}_{\text {boost }}$ comprised the gross tumor with a $2-3 \mathrm{~cm}$

Table 1 Patient characteristics

\begin{tabular}{ll}
\hline Characteristics & \\
\hline Number of patients & 34 \\
\hline Median age (range) & $59(29-79)$ \\
Sex & $25(73.5 \%)$ \\
$\quad$ Male & $9(26.5 \%)$ \\
$\quad$ Female & \\
Clinical T stage & $1(2.9 \%)$ \\
T1-2 & $33(97.1 \%)$ \\
$\quad$ T3-4 & \\
Clinical N stage & $3(8.8 \%)$ \\
$\quad \mathrm{N}-$ & $31(91.2 \%)$ \\
$\quad \mathrm{N}+$ & \\
CEA (ng/mL) & $17(50 \%)$ \\
$\quad<5.0$ & $17(50 \%)$ \\
$\quad \geq 5.0$ &
\end{tabular}

margin. The planning target volume (PTV) received a dose of $45 \mathrm{~Gy}$ and the PTV boost received a dose of $50 \mathrm{~Gy}$ in 25 fractions simultaneously, using intensitymodulated radiotherapy (IMRT) technique. The median PTV was 1617 (range, 859 to 2166) cc. Treatment plans were generated on a Pinnacle Treatment Planning System (v 9.8), using 6-MV or 10-MV photons. All patients were treated on a Siemens Oncor Impression Plus accelerator. Capecitabine was administered at a dose of 1650 $\mathrm{mg} / \mathrm{m}^{2} /$ day during $\mathrm{nCRT}$.

\section{Surgery and postoperative chemotherapy}

Surgery was performed 6-10 weeks after the completion date of nCRT. After the operation, postoperative chemotherapy was administered. The postoperative chemotherapy comprised 5-8 (median 7) cycles of FOLFOX (a regimen of 5-FU, leucovorin, and oxaliplatin every 2 weeks), 4-6 (median 5) cycles of XELOX (a regimen of capecitabine and oxaliplatin every 3 weeks), or 4-6 (median 5) cycles of capecitabine monotherapy (every 3 weeks).

\section{ALCs during treatment}

Prior to treatment (defined as W0), 24 patients $(70.59 \%)$ had normal ALC, 10 patients (29.41\%) experienced grade 1 lymphopenia, and no patient had grade $2-4$ lymphopenia. Before operation, two patients $(5.88 \%)$ had normal ALC, six patients (17.65\%) had grade 1 lymphopenia, 22 patients (64.71\%) had grade 2 lymphopenia, four patients (11.76\%) had grade 3 lymphopenia, and no patient had grade 4 lymphopenia. The median ALC at baseline (W0) was 1.62 (range, 1.14-4.36) $\times 10^{9}$ cells $/ \mathrm{L}$. The median ALCs declined to 1.10 (range, 0.63-2.02), 0.76 (range, 0.45-1.87), 0.59 (range, 0.39-1.36), 0.50 (range, 0.321.27), and 0.61 (range, $0.31-1.08$ ) $\times 10^{9}$ cells/L from week 1 (W1) to week 5 (W5) during CRT, respectively. The median pre-SL was 0.76 (range, $0.32-2.32$ ) $\times 10^{9}$ cells/L. Significant differences were found between ALCs at W0 and W1 $(p=0.041), \mathrm{W} 0$ and W2 $(p<0.001)$, W0 and W3 $(p<0.001), \mathrm{W} 0$ and W4 $(p<0.001)$, W0 and W5 $(p<0.001), \mathrm{W} 1$ and W2 $(p=0.020)$, and W5 and pre-SL $(p=0.010)$. However, no significant difference was detected between ALCs at W2 and W3 $(p=0.056)$, W3 and W4 $(p=0.474)$, and W4 and W5 $(p=0.973$, detailed in Fig. 1).

\section{Clinical and treatment characteristics associated with DFS}

The DFS ranged from 4 to 70 months and the median DFS of all patients had not been reached through the last follow-up date of December 31, 2018. Twentyseven patients were alive while 7 patients died of disease progression. The median follow-up time of the surviving patients was 32 months. Twelve patients had progressive disease. Ten of them had distant 


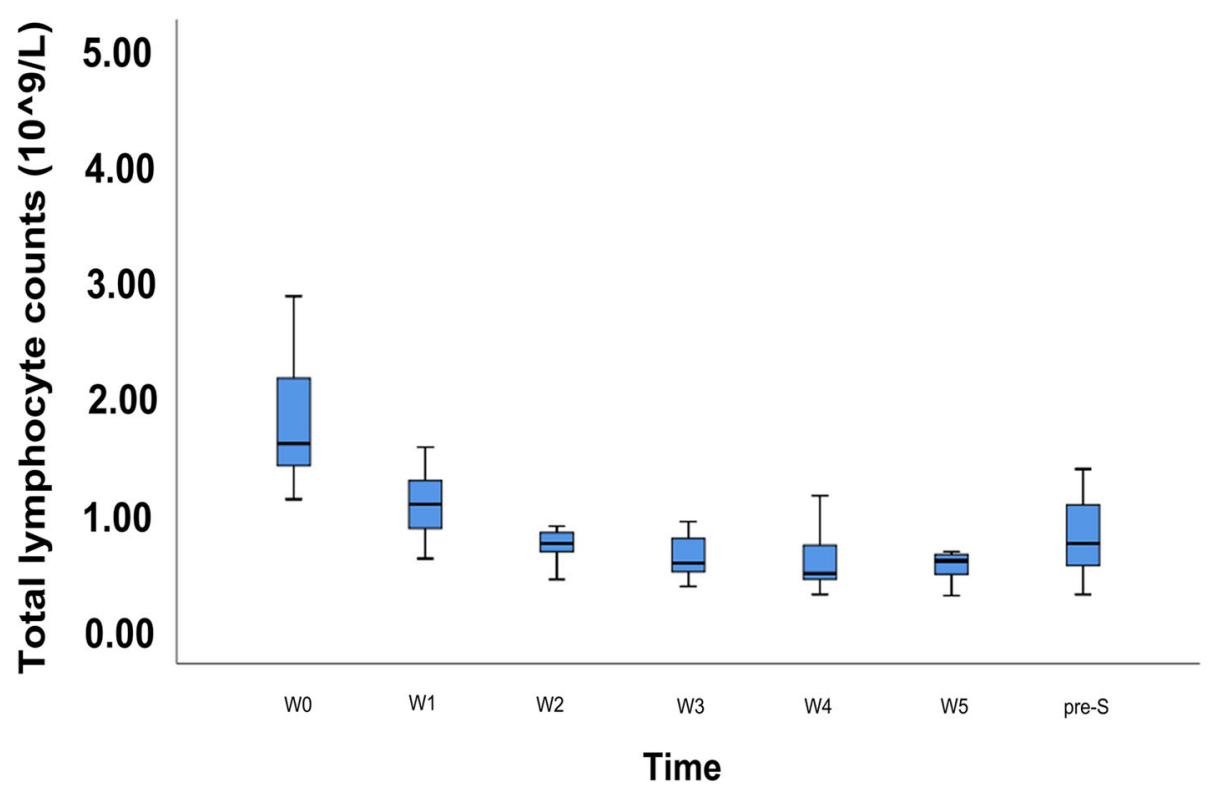

Fig. 1 Absolute lymphocyte counts (ALCS) changed over time. Significant differences were found between ALCs at W0 and W1 ( $p=0.041$ ), W0 and W2 $(p<0.001)$, W0 and W3 $(p<0.001)$, W0 and W4 $(p<0.001)$, and W0 and W5 ( $p<0.001)$. W0: pre-RT; W1-5: week 1 to week 5 during CRT; pre-S: before operation

metastases, one had a local recurrence, and one had both. Pre-SLR was the only prognostic factor (Table 2) with significant associations with DFS in both univariate $(\mathrm{HR}=6.287 ; 95 \%$ confidence interval $[\mathrm{CI}], 1.374-$ 28.781; $p=0.018)$ and multivariate analyses $(\mathrm{HR}=$ 7.347; 95\% CI, 1.595-33.850; $p=0.011$ ). No other factor was significantly associated with DFS, including age, sex, CEA, downstage, positive lymph node, pre-RTL, pre-SL, lymphocyte nadir, and PTV. Figure 2 shows the OS and DFS curves of the whole cohort and Fig. 3 shows Kaplan-Meier DFS curves stratified by pre-SLR. The median DFS was 28 months in patients with a low
pre-SLR and the median DFS had not been reached in patients with a high pre-SLR $(p=0.007)$.

\section{Patient characteristics and treatment factors associated with pre-SLR}

Table 3 shows that PBM V30 (median 47.18\%; range 28.01-59.14\%) was the only predictive factor for preSLR in both univariate and multivariate regression analyses. PBM V20, PBM V40, PBM $\mathrm{D}_{\text {mean }}$, and PTV were not significantly associated with pre-SLR in univariate analysis or multivariate analysis (Table 3 ).

Table 2 Univariate and multivariate analysis for variables associated with DFS

\begin{tabular}{|c|c|c|c|c|c|c|c|}
\hline \multirow[t]{2}{*}{ Clinical factors } & & \multicolumn{3}{|c|}{ Univariate analysis } & \multicolumn{3}{|c|}{ Multivariable analysis } \\
\hline & & $\mathrm{HR}$ & $95 \% \mathrm{Cl}$ & $p$ & $\mathrm{HR}$ & $95 \% \mathrm{Cl}$ & $p$ \\
\hline Age & $(\geq 59$ vs< 59$)$ & 0.935 & $0.338-3.255$ & 1.048 & & & \\
\hline Sex & (F vs $M)$ & 0.428 & $0.093-1.980$ & 0.278 & & & \\
\hline CEA & $(\geq 5.0$ vs $<5.0)$ & 0.980 & $0.315-3.049$ & 0.972 & - & & \\
\hline Downstage & (n vs y) & 2.104 & $0.459-9.653$ & 0.338 & & & \\
\hline $\mathrm{N}$ status & $(\mathrm{N}+$ vs $\mathrm{N}-)$ & 1.924 & $0.610-6.07$ & 0.264 & & & \\
\hline Pre-SL & $(\mathrm{L}$ vs H) & 3.363 & $0.987-13.656$ & 0.052 & & & \\
\hline Pre-RTL & $(\mathrm{L}$ vs H) & 0.433 & $0.095-1.983$ & 0.281 & & & \\
\hline Lnadir & $(\mathrm{L}$ vs H) & 1.804 & $0.542-6.003$ & 0.336 & & & \\
\hline Pre-SLR & $(\mathrm{L}$ vs H) & 6.287 & $1.374-28.781$ & 0.018 & 7.347 & $1.595-33.850$ & 0.011 \\
\hline
\end{tabular}

$H R$, hazard ratio; $C l$, confidence interval; $F$, female; $M$, male; $C E A$, carcino-embryonic antigen; $N$, lymph node; $N+$, lymph node positive; $N-$, lymph node negative; pre-SL, absolute lymphocyte counts before surgery; pre- $R T L$, absolute lymphocyte counts before concurrent chemoradiotherapy; $L$ nadir, the lowest absolute lymphocyte count during concurrent chemoradiotherapy; $n$, no; $y, y e s ; L$, low; $H$, high 

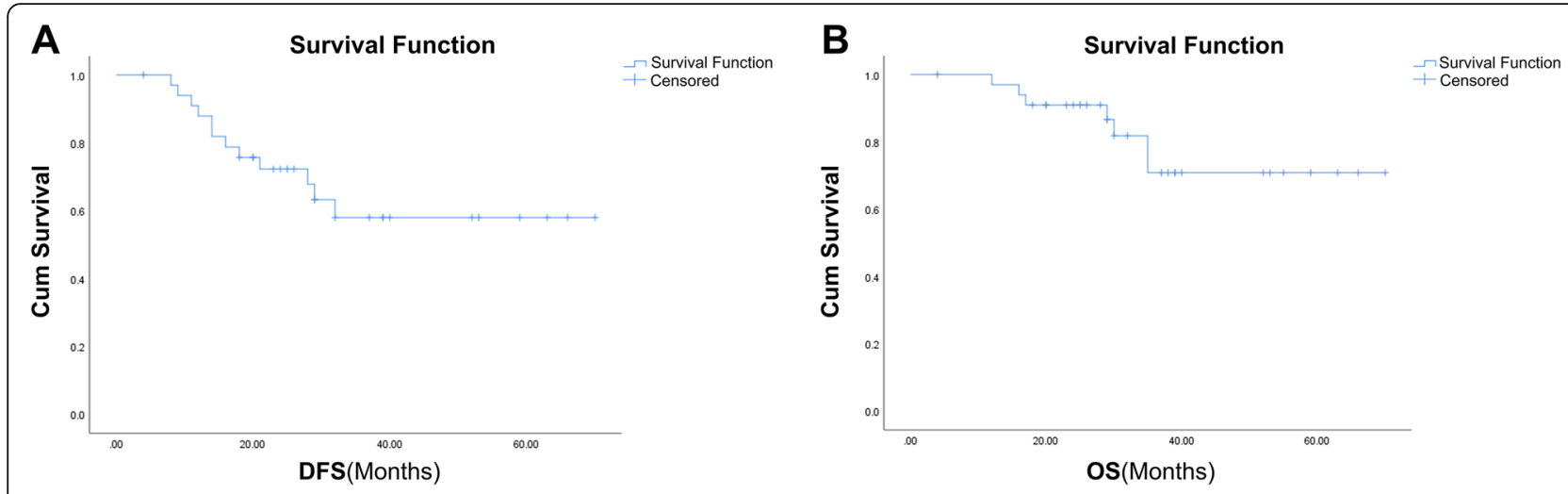

Fig. 2 Disease-free survival (DFS) and overall survival (OS) in the whole patient cohort

Patient characteristics and treatment-related factors associated with downstaging

Neither patient characteristics nor treatment-related factors were related to downstaging, including pre-RTL, ALC nadir, pre-SLR, sex, age, CEA, and PTV. Furthermore, none of these factors was related to positive lymph node status.

\section{Discussion}

It is widely accepted that the host anti-tumor immune response is important in patients with cancer. The association between circulating lymphocyte count and DFS has not been well documented in locally advanced rectal cancer. In the present study, we reported that circulating
ALCs declined significantly during nCRT and that preSLR was the only prognostic factor for DFS in rectal cancer. The V30 of the pelvic bone marrow was further found to be the only predictive factor inversely associated with a high pre-SLR.

To our knowledge, this is the first study to report the association between pre-SLR and DFS in patients with locally advanced rectal cancer receiving nCRT followed by TME. Heo and coauthors reported that a sustained lymphocyte ratio in the fourth week of preoperative CRT was a predictor for pathologic complete response in rectal cancer [8]. However, survival data were not available in that study. Another study reported the initial lymphocyte ratio to be a

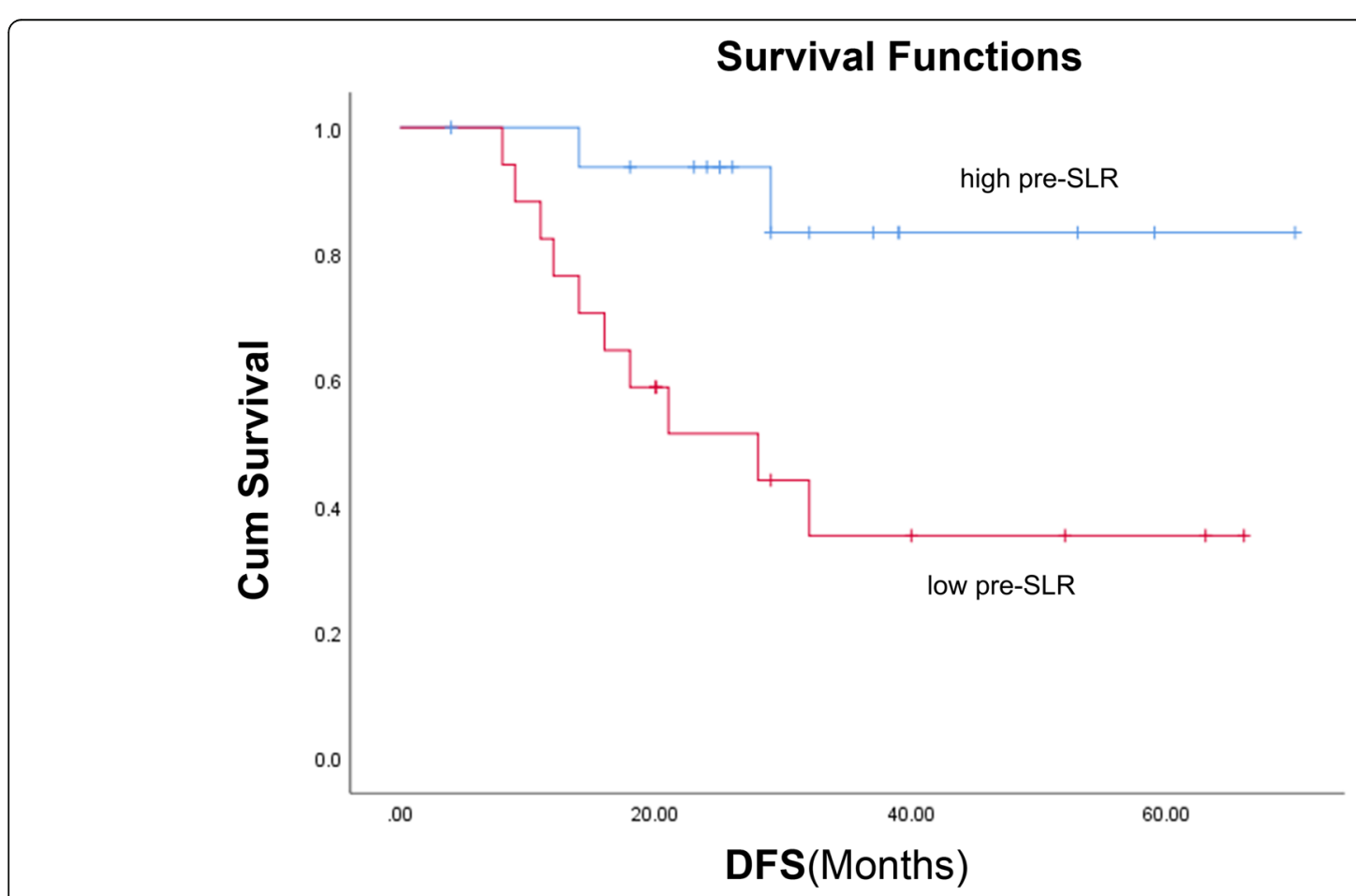

Fig. 3 DFS curves stratified by pre-SLR level 
Table 3 Univariate and multivariate analysis for variables associated with pre-SLR

\begin{tabular}{|c|c|c|c|c|c|c|c|}
\hline & \multicolumn{3}{|c|}{ Univariate analysis } & \multicolumn{3}{|c|}{ Multivariable analysis } \\
\hline \multicolumn{2}{|c|}{ Clinical tactors } & \multirow{2}{*}{$\begin{array}{l}\mathrm{HR} \\
1.257\end{array}$} & \multirow{2}{*}{$\begin{array}{l}95 \% \mathrm{Cl} \\
0.333-4.742\end{array}$} & \multirow{2}{*}{$\frac{p}{0.735}$} & \multirow[t]{2}{*}{$\mathrm{HR}$} & \multirow[t]{2}{*}{$95 \% \mathrm{Cl}$} & \multirow[t]{2}{*}{$p$} \\
\hline Age & $(\leq 60$ vs $>60)$ & & & & & & \\
\hline Sex & (F vs $M)$ & 2.062 & $0.492-8.654$ & 0.322 & & & \\
\hline $\mathrm{N}$ status & $(\mathrm{N}+\mathrm{vs} \mathrm{N}-)$ & 1.250 & $0.299-5.230$ & 0.760 & & & \\
\hline CEA & $(>5.0$ vs $\leq 5.0)$ & 0.606 & $0.158-2.319$ & 0.464 & & & \\
\hline Pre-RTL & (L vs H) & 8.000 & $1.686-37.981$ & 0.009 & & & \\
\hline V5 & (L vs H) & 4.469 & $1.054-18.938$ & 0.042 & & & \\
\hline V10 & (L vs H) & 2.041 & $0.521-7.999$ & 0.306 & & & \\
\hline V20 & $(\mathrm{L}$ vs $H)$ & 3.361 & $0.823-13.722$ & 0.091 & & & \\
\hline V30 & (L vs H) & 5.760 & $1.317-25.187$ & 0.020 & 5.760 & $1.317-25.187$ & 0.020 \\
\hline V40 & (L vs H) & 3.361 & $0.823-13.722$ & 0.091 & & & \\
\hline Dmean & (L vs H) & 3.361 & $0.823-13.722$ & 0.091 & & & \\
\hline
\end{tabular}

$H R$, hazard ratio; $C l$, confidence interval; $F$, female; $M$, male; $C E A$, carcino-embryonic antigen; $N$, lymph node; $N+$, lymph node positive; $N-$, lymph node negtive; pre-SL, absolute lymphocyte counts before surgery; pre- $R T L$, absolute lymphocyte counts before concurrent chemoradiotherapy; $n$, no; $y$, yes; $L$, low; $H$, high

predictor of pathologic complete response in rectal cancer. In addition, lymphocyte ratio was also a predictor of DFS [7]. Treatment-related lymphopenia has been verified to be associated with prognosis in other cancers. Tang et al. found that lower ALC after definitive radiotherapy was associated with shorter OS and event-free survival in non-small lung cancer [10]. Cho and coauthors verified that lymphocyte nadir was a prognostic factor in limited-stage small-cell lung cancer [11]. Neither pre-RTL nor pre-SL was associated with DFS in the present study. It is possible that the patient number was too low to observe an association between ALCs and DFS. However, a high preSLR was a useful predictor for better DFS. Therefore, pre-SLR may be a better prognostic factor than preRTL and pre-SL because it considers both pre-CRT and post-CRT ALCs.

We also found that circulating ALCs declined drastically during nCRT, a finding in accordance with previous studies and has been suggested to be due to lymphocyte sensitivity to radiotherapy [7, 8, 10-12]. This finding further indicates the importance of sustaining lymphocyte number during CRT in routine clinical practice. Circulating lymphocyte numbers are easily affected by various factors. However, none of the clinical characteristics in the present study, including age, sex, lymph node status, and CEA, were associated with the pre-SLR.

We further found that the V30 of the PBM significantly affected the pre-SLR. The irradiation dose to the PBM is reported to be significantly associated with hematologic toxicity in rectal cancer [13, 14]. Similar results were reported in cervical and anal cancer [15-19]. However, the influence of radiotherapy dose to the PBM on lymphocyte counts was not described. Our results indicate that the irradiation dose to the PBM may be related to lymphocyte counts. Limiting the irradiation dose to the PBM might help to preserve ALCs. We will initiate a clinical trial to investigate whether bone marrow-sparing IMRT can help rectal cancer patients to sustain higher pre-SLR levels.

Peripheral lymphocyte counts and lymphocyte subtypes were also predictors of favorable response after nCRT in rectal cancer [7, 8]. However, no clinical factors were associated with downstage or positive lymph node in the present study, including pre-RTL, ALC nadir, pre-SLR, sex, age, CEA, and PTV volume. Many predictive factors for pathological responses have been reported in rectal cancer, including CEA, distance from the anal verge [20], p21, and other factors [21]. However, currently, there is no widely accepted predictive factor. Therefore, it is reasonable that our results were not concordant with those of previous studies regarding the predictive factors for pathological downstaging.

There are several limitations in the present study. First, bias is possible because it is a small single-center retrospective study. Our results should be verified in larger prospective studies. Second, lymphocyte subpopulations were not examined and we did not know the lymphocyte subtypes associated with DFS. The changes in lymphocyte subpopulation should be assessed in future studies. Third, we used the entire pelvic bone to represent the pelvic bone marrow and more precise methods to evaluate active bone marrow such as positron emission tomography-computed tomography (PET-CT) were not considered. However, pelvic bone delineation was easy to perform, had a low cost and was time-saving. 


\section{Conclusion}

The present study suggests that pre-SLR may be a useful prognostic factor for DFS in patients with rectal cancer receiving $\mathrm{nCRT}$ and TME. The V30 of PBM was a useful predictive factor for the level of pre-SLR. Further study is warranted on preserving pre-SLR with bone marrowsparing IMRT in patients with locally advanced rectal cancer.

\section{Abbreviations}

nCRT: Neoadjuvant concurrent chemoradiotherapy; ALCs: Absolute lymphocyte counts; pre-SLR: Pre-SL to pre-RTL ratio; HR: Hazard ratio; Cl: Confidence interval; PBM: Pelvic bone marrow; DFS: Disease-free survival; OS: Overall survival; CEA: Carcino-embryonic antigen; TME: Total mesorectal excision; CTV: Clinical target volume; PTV: Planning target volume

\section{Acknowledgements}

Not applicable.

\section{Authors' contributions}

JT, MZ and HZ drafted the manuscript. HX and GY participated in the design of the study and performed the statistical analysis. TS, YJ and JY conceived the study and participated in its design and coordination. XL revised the manuscript. All authors read and approved the final manuscript.

\section{Funding}

This study was supported by the Zhejiang Province Medical and Health Care Project (2019KY279) and the Zhejiang Provincial Natural Science Foundation of China (LY18H160035)

\section{Availability of data and materials}

The data and materials in the current study are available from the corresponding author on reasonable request.

\section{Ethics approval and consent to participate}

This study was conducted in accordance with the Helsinki Declaration II and was approved by the Institutional Review Boards of Zhejiang Provincial People's Hospital and Taizhou cancer Hospital.

\section{Consent for publication}

Not applicable.

\section{Competing interests}

The authors declare that they have no competing interests.

\section{Author details}

${ }^{1}$ Department of Radiation Oncology, Cancer Center, Zhejiang Provincial People's Hospital, People's Hospital of Hangzhou Medical College, No. 158, Shangtang Road, Xiacheng District, Hangzhou 310014, China. ${ }^{2}$ Department of Radiation Oncology, Taizhou Cancer Hospital, Taizhou 317502, China. ${ }^{3}$ Graduate Department, Bengbu Medical College, No. 2600 Donghai Avenue, Bengbu 233000, China.

Received: 16 July 2019 Accepted: 8 November 2019

Published online: 30 November 2019

\section{References}

1. Bray F, Ferlay J, Soerjomataram I, et al. Global cancer statistics 2018: GLOBOCAN estimates of incidence and mortality worldwide for 36 cancers in 185 countries. CA Cancer J Clin. 2018, 68(6):394-424.

2. Sauer R, Liersch T, Merkel $\mathrm{S}$, et al. Preoperative versus postoperative chemoradiotherapy for locally advanced rectal cancer: results of the German CAO/ARO/AIO-94 randomized phase III trial after a median followup of 11 years. J Clin Oncol. 2012;30:1926-33.

3. Roh MS, Colangelo LH, O'Connell MJ, et al. Preoperative multimodality therapy improves disease-free survival in patients with carcinoma of the rectum: NSABP R-03. J Clin Oncol. 2009;27:5124-30.
4. Rozek LS, Schmit SL, Greenson JK, et al. Tumor-infiltrating lymphocytes, Crohn's-like lymphoid reaction, and survival from colorectal cancer. J Natl Cancer Inst. 2016;108(8). https://doi.org/10.1093/jnci/djw027.

5. Ohtani H. Focus on TILs: prognostic significance of tumor infiltrating lymphocytes in human colorectal cancer. Cancer Immun. 2007;7:4.

6. Venkatesulu BP, Mallick S, Lin SH, Krishnan S. A systematic review of the influence of radiation-induced lymphopenia on survival outcomes in solid tumors. Crit Rev Oncol Hematol. 2018;123:42-51.

7. Kitayama J, Yasuda K, Kawai K, et al. Circulating lymphocyte is an important determinant of the effectiveness of preoperative radiotherapy in advanced rectal cancer. BMC Cancer. 2011;11:64.

8. Heo J, Chun M, Noh OK, et al. Sustaining blood lymphocyte count during preoperative chemoradiotherapy as a predictive marker for pathologic complete response in locally advanced rectal cancer. Cancer Res Treat. 2016:48:232-9.

9. Ceze N, Thibault G, Goujon G, et al. Pre-treatment lymphopenia as a prognostic biomarker in colorectal cancer patients receiving chemotherapy. Cancer Chemother Pharmacol. 2011;68:1305-13.

10. Tang C, Liao Z, Gomez D, et al. Lymphopenia association with gross tumor volume and lung V5 and its effects on non-small cell lung cancer patient outcomes. Int J Radiat Oncol Biol Phys. 2014;89:1084-91.

11. Cho O, Oh YT, Chun M, et al. Radiation-related lymphopenia as a new prognostic factor in limited-stage small cell lung cancer. Tumour Biol. 2016;37:971-8.

12. Balmanoukian A, Ye $X$, Herman J, et al. The association between treatmentrelated lymphopenia and survival in newly diagnosed patients with resected adenocarcinoma of the pancreas. Cancer Invest. 2012;30:571-6.

13. Wan J, Liu K, Li K, et al. Can dosimetric parameters predict acute hematologic toxicity in rectal cancer patients treated with intensitymodulated pelvic radiotherapy? Radiat Oncol. 2015;10:162.

14. Jianyang W, Yuan T, Yuan T, et al. A prospective phase II study of magnetic resonance imaging guided hematopoietical bone marrow-sparing intensitymodulated radiotherapy with concurrent chemotherapy for rectal cancer. Radiol Med. 2016:121:308-14.

15. Mell LK, Schomas DA, Salama JK, et al. Association between bone marrow dosimetric parameters and acute hematologic toxicity in anal cancer patients treated with concurrent chemotherapy and intensity-modulated radiotherapy, Int J Radiat Oncol Biol Phys. 2008:70:1431-7.

16. Rattan R, Kapoor R, Bahl A, et al. Comparison of bone marrow sparing intensity modulated radiotherapy (IMRT) and three-dimensional conformal radiotherapy (3DCRT) in carcinoma of anal canal: a prospective study. Ann Transl Med. 2016:4:70.

17. Franco P, Ragona R, Arcadipane F, et al. Dosimetric predictors of acute hematologic toxicity during concurrent intensity-modulated radiotherapy and chemotherapy for anal cancer. Clin Transl Oncol. 2017;19:67-75.

18. Rose BS, Liang Y, Lau SK, et al. Correlation between radiation dose to (1)(8)F-FDG-PET defined active bone marrow subregions and acute hematologic toxicity in cervical cancer patients treated with chemoradiotherapy. Int J Radiat Oncol Biol Phys. 2012:83:1185-91.

19. Albuquerque K, Giangreco D, Morrison C, et al. Radiation-related predictors of hematologic toxicity after concurrent chemoradiation for cervical cancer and implications for bone marrow-sparing pelvic IMRT. Int J Radiat Oncol Biol Phys. 2011;79:1043-7.

20. Restivo A, Zorcolo L, Cocco IM, et al. Elevated CEA levels and low distance of the tumor from the anal verge are predictors of incomplete response to chemoradiation in patients with rectal cancer. Ann Surg Oncol. 2013;20:864-71.

21. Bertolini F, Bengala C, Losi L, et al. Prognostic and predictive value of baseline and posttreatment molecular marker expression in locally advanced rectal cancer treated with neoadjuvant chemoradiotherapy. Int J Radiat Oncol Biol Phys. 2007;68:1455-61.

\section{Publisher's Note}

Springer Nature remains neutral with regard to jurisdictional claims in published maps and institutional affiliations. 